\title{
Whole-liver histogram analysis of blood oxygen level-dependent functional magnetic resonance imaging in evaluating hepatic fibrosis
}

\author{
Hai-Feng Liu", Qing Wang", Ya-Nan Du, Jiu-Le Ding, Jing-Gang Zhang, Wei Xing \\ Department of Radiology, Third Affiliated Hospital of Soochow University \& Changzhou First People's Hospital, Changzhou, China \\ Contributions: (I) Conception and design: HF Liu, W Xing; (II) Administrative support: W Xing; (III) Provision of study materials or patients: \\ HF Liu, Q Wang, YN Du; (IV) Collection and assembly of data: HF Liu, Q Wang; (V) Data analysis and interpretation: HF Liu, Q Wang; (VI) \\ Manuscript writing: All authors; (VII) Final approval of manuscript: All authors. \\ \#These authors contributed equally in this work. \\ Correspondence to: Wei Xing. No. 185, Juqian Street, Tianning District, Changzhou 213003, China. Email: suzhxingwei@suda.edu.cn.
}

\begin{abstract}
Background: To explore whole-liver histogram analysis (HA) with blood oxygen level-dependent functional magnetic resonance imaging (BOLD-fMRI) in evaluating and diagnosing hepatic fibrosis (HF) in a CCl4-induced rabbit model.
\end{abstract}

Methods: One hundred rabbits were classified as CCl4-induced HF groups ( $\mathrm{n}=80)$ and control group ( $\mathrm{n}=20)$, and were scheduled for BOLD-fMRI examination on a $3.0 \mathrm{~T}$ scanner. Whole-liver HA metrics, including the mean, median, skewness, kurtosis, inhomogeneity, entropy, and $n^{\text {th }}$ percentiles, were extracted from the $\mathrm{R}_{2}{ }^{*}$ map. Parameters were compared among the different HF stages using receivers operating characteristic analysis and Mann-Whitney U-test.

Results: In all, 17, 18, 19, 17, and 15 rabbits were pathologically diagnosed as F0, F1, F2, F3, and F4, respectively. HA parameters, including the median, inhomogeneity, entropy, and the $75^{\text {th }}$ and $90^{\text {th }}$ percentiles of the BOLD $\mathrm{R}_{2}{ }^{*}$ map, demonstrated significant positive correlations with the fibrosis stage ( $\mathrm{r}=0.226-0.718$, $\mathrm{P} \leq 0.039)$. The $75^{\text {th }}$ percentiles demonstrated higher diagnostic efficacy than the other HA parameters in fibrosis staging, with an AUC value of 0.86 for $\geq F 1,0.87$ for $\geq F 2,0.87$ for $\geq F 3$, and 0.86 for $\geq F 4$.

Conclusions: BOLD-fMRI HA provides increased diagnostic performance in staging HF, especially for the $75^{\text {th }}$ percentiles.

Keywords: Hepatic fibrosis (HF); blood oxygen level-dependent (BOLD); histogram analysis (HA); whole liver

Submitted Sep 02, 2020. Accepted for publication Nov 30, 2020.

doi: $10.21037 /$ apm-20-1753

View this article at: http://dx.doi.org/10.21037/apm-20-1753

\section{Introduction}

Hepatic fibrosis (HF) is caused by the accumulation of extracellular matrix proteins and is a common histopathological feature of chronic liver disease resulting from various hepatic, metabolic and cholestatic diseases $(1,2)$. HF represents the critical prestage of liver cirrhosis and may eventually deteriorate into hepatocellular carcinoma in the absence of antifibrotic therapy in earlystage HF or liver transplantation in advanced HF stages
(3-5). Therefore, precise evaluation of the HF stage is not only important for diagnosis, but it is also increasingly important for establishing prognosis and guiding subsequent treatment.

Although liver biopsy is considered to be the current gold standard for diagnosing and staging HF, it has serious limitations including sampling error as high as $25 \%$, high intra/interobserver variability, and invasive complications such as pain in $25-40 \%$ of patients and bleeding or death 
in $0.3-0.6 \%$ of patients $(6,7)$. Serum markers provide noninvasive assessment of $\mathrm{HF}$, but a newly published metaanalysis of 32 studies and 11,179 patients demonstrated a low sensitivity of $69 \%$ (95\% CI: $63-73 \%$ ) and specificity of $71 \%$ (95\% CI: $66-75 \%$ ) for the fibrosis-4 index (FIB-4) in distinguishing HF stages, revealing that serological testing was inadequate in precisely discriminating HF (8). Thus, in recent decades, studies have been increasingly aimed at investigating noninvasive and accurate imaging techniques for assessing HF.

Blood oxygen level-dependent functional magnetic resonance imaging (BOLD-fMRI) using deoxyhemoglobin as an endogenous contrast mechanism is the only noninvasive imaging method that reflects the blood oxygen level in vivo and can be used to assess changes in blood flow and oxygenation $(9,10)$. Ning et al. (11) suggested that the signal intensity of BOLD-fMRI was of great importance in discriminating the HF stage $(\mathrm{P}<0.05)$ and demonstrating a strong negative correlation with $\mathrm{HF}$ progression $(\mathrm{r}=0.773, \mathrm{P}<0.05)$. Histogram analysis $(\mathrm{HA})$ is a new image postprocessing approach which employs mathematical analysis to evaluate variations in the grayscale intensity of images that are not detected by the human eye. Previous studies indicated that HA with different functional MR sequences including diffusion-weighted imaging (DWI) (12), susceptibility-weighted imaging (SWI) (13), and MR elastography (MRE) (14) were valuable for reflecting histopathological heterogeneity and diagnosing HF stages. However, no well-validated study has been conducted to assess the value of whole-liver HA with BOLD-fMRI in diagnosing and staging HF.

Therefore, this study aimed to explore whole-liver HA based on BOLD-fMRI in quantifying the grayscale distribution and staging HF in a rabbit model induced by carbon tetrachloride with histopathological results as a reference standard. We present the following article in accordance with the ARRIVE reporting checklist (available at http://dx.doi.org/10.21037/apm-20-1753).

\section{Methods}

\section{Animal model}

Experiments were performed under a project license (No. 2019-022) granted by institutional ethics board of Third Affiliated Hospital of Soochow University, in compliance with NIH guidelines for the care and use of animals. One hundred healthy female New Zealand white rabbits weighing 2.0-2.5 $\mathrm{kg}$ and aged 6 months old were included. According to the method of block randomization, these rabbits were randomly divided into the HF group $(\mathrm{n}=80)$, in which $\mathrm{HF}$ was induced by a subcutaneous injection of a $50 \% \mathrm{CCl}_{4}$ oil solution for 4-16 weeks, and a normal control group $(n=20)$, in which the rabbits received subcutaneous injections of normal saline solution at the same dosage and frequency (15). The dose of $\mathrm{CCl}_{4}$ increased gradually as follows: $0.1 \mathrm{~mL} / \mathrm{kg}$ from week 1 to week $3,0.2 \mathrm{~mL} / \mathrm{kg}$ from week 4 to week 6 , and $0.3 \mathrm{~mL} / \mathrm{kg}$ from week 7 to week 16 .

\section{BOLD-fMRI protocol}

At the end of the 4th, 5th, 6th, and 15 th weeks after the first injection of $\mathrm{CCl}_{4}, 20$ rabbits in the HF group and 5 rabbits in the control group underwent BOLD imaging with an 18-channel HeadNeck coil on a 3.0 T MRI scanner (Signa HDx, GE Healthcare, Waukesha, WI). Rabbits were fasted for 12 hours, and then general anesthesia was induced by intramuscular injection of $0.1 \mathrm{~mL} / \mathrm{kg}$ xylazine before the examination, followed by mechanical ventilation anesthesia using a small animal anesthesia instrument (ABS100, Y100141035, China) with the anesthesia level of 3.5\% isoflurane (isoflurane, USP, China) for rabbits during MRI scanning.

The rabbits were placed in a supine position with the head positioned straight forward and fixed with an abdominal elastic belt to decrease respiratory movement. The BOLD-fMRI protocol included the two following sequences: (I) transversal BOLD-fMRI: BOLD images were acquired using a multiple gradient recalled echo sequence with the following parameters: $\mathrm{TR}=1,090 \mathrm{~ms}$, TE $=3.4$ $39.2 \mathrm{~ms}, \mathrm{FA}=15^{\circ}, \mathrm{FOV}=160 \times 140 \mathrm{~mm}$, matrix $=218 \times 256$, $\mathrm{ST}=4 \mathrm{~mm}, \mathrm{SP}=0.4 \mathrm{~mm}, \mathrm{Nex}=2$, bandwidth $=331 \mathrm{~Hz} /$ pixel, acquisition time $=2$ min 10 s. (II) Precontrast axial T1weighted imaging (T1WI) with turbo-spin-echo sequence.

\section{BOLD image analysis}

The BOLD images were analyzed by consensus by two abdominal radiologists (HFL and QW) with 5 and 10 years of experience, respectively, who were blinded to the histopathological results.

After BOLD-fMRI scanning, the original $\mathrm{R}_{2}{ }^{*}$ maps were generated and stored in digital imaging and communications in medicine (DICOM) format, and postprocessed using Firevoxel software (FireVoxel, 329; https://wp.nyu.edu/ 
firevoxel/downloads/) for performing HA. ROIs were manually drawn on each transversal image covering the whole liver parenchyma by two mentioned radiologists, avoiding visible vessels, bile ducts, artifacts, and liver edges. Then, an $\mathrm{R}_{2}{ }^{*}$ histogram and frequency table were generated for the whole-liver ROIs, and the following data were calculated: the mean, median, inhomogeneity, skewness, kurtosis, entropy, $10^{\text {th }}, 25^{\text {th }}, 75^{\text {th }}$, and $90^{\text {th }}$ percentiles. (I) Inhomogeneity is thought to represent the variation divided by the mean gray-level intensity across all pixels; (II) the skewness and kurtosis represent the asymmetry and peakedness of the histogram, respectively; (III) entropy reveals the irregular distribution within the histogram; and (IV) the $n^{\text {th }}$ percentile indicates the $n \%$ of the voxel values from the histogram, which are found to the left.

\section{Histopathological examination}

These experimental rabbits were sacrificed immediately through aeroembolism after satisfactorily completing BOLD-fMRI. The liver was harvested and fixed in $10 \%$ formalin and stained with Masson's trichrome stain. Then, the stained slices were evaluated by two well-trained pathologists who were unaware of the BOLD images, and discrepancies were solved in consensus. The HF degree was classified according to the METAVIR system, and it was divided into five stages: $\mathrm{F} 0$, no fibrosis; F1, portal fibrosis without septa; F2, portal fibrosis and a few septa; F3, numerous septa without cirrhosis; and F4, cirrhosis.

\section{Statistical analysis}

A Shapiro-Wilk test was used to evaluate the normal distribution of the HA parameters. If those parameters were determined to be normally distributed, the data are presented as the mean $\pm \mathrm{SD}$. Correlations between parameters and HF stages were evaluated using Spearman's correlation coefficient ( $\mathrm{r}$ ), and a $\mathrm{P}$ value $<0.05$ was considered statically significant. Pairwise comparisons of histogram parameters with different HF stages were tested through the Mann-Whitney U-test with Bonferroni correction, and a two-sided $\mathrm{P}$ value $<0.05$ was considered statistically significant. For significant parameters (significant correlation with HF progression), box plots of each parameter were plotted for every HF stage, and the receiver operating characteristic (ROC) curve with area under ROC (AUC) was further determined to evaluate diagnostic values in differentiating $\mathrm{F} 0 v s . \geq \mathrm{F} 1$ (F1-F4), F0$\mathrm{F} 1 v s . \geq \mathrm{F} 2$ (F2-F4), F0-F2 vs. $\geq \mathrm{F} 3$ (F3-F4), and F0-F3 vs. $\geq$ F4 (F4), respectively. The optimal cutoff values of each metric for identifying different HF stages were determined by the maximum Youden index (sensitivity + specificity -1 ). $\mathrm{P}<0.05$ was considered statistically significant. All statistical analyses were performed through SPSS (version 22.0, Chicago, IL) and MedCalc (version 18.2.1, Acacialaan, Belgium) software.

\section{Results}

\section{Animal model and bistopathological results}

Of the 100 rabbits, 1 normal rabbit and $7 \mathrm{HF}$ rabbits died owing to poor tolerance of anesthesia or $\mathrm{CCl}_{4}$ administration, and 2 rabbits in the control group and 4 in the HF group were excluded result from severe breathing and movement artifacts. In all, 17 normal rabbits and $69 \mathrm{HF}$ rabbits were finally enrolled, with 17 rabbits in stage F0, 18 rabbits in stage $\mathrm{F} 1,19$ rabbits in stage $\mathrm{F} 2,17$ rabbits in stage $\mathrm{F} 3$, and 15 rabbits in stage $\mathrm{F} 4$, respectively.

\section{$H A$ parameters among the different $H F$ stages}

Representative images of Masson's trichrome-stained samples, schematic drawings of the ROI, rainbow $\mathrm{R}_{2}{ }^{*}$ maps, and histogram images for stage $\mathrm{F} 2$ in the rabbit liver are shown in Figure 1. The results of the Shapiro-Wilk test suggested that all quantitative parameters were normally distributed, which shown in Table 1.

\section{Correlations between HA parameters and HF stages}

The spearman's correlation results (Table 2) and box plots (Figure 2) indicated a strong positive correlation between the $75^{\text {th }}$ and HF stage $(\mathrm{r}=0.718, \mathrm{P}<0.001)$, whereas there were moderate positive correlations for the HA parameters including the $90^{\text {th }}(\mathrm{r}=0.670)$, inhomogeneity $(\mathrm{r}=0.644)$, entropy $(\mathrm{r}=0.318)$, and median $(\mathrm{r}=0.226)$ with increasing HF stage $(\mathrm{P}<0.05)$. However, no statistical correlations were found between the mean, 10th, 25th, skewness, and kurtosis with HF stage progression.

\section{Differences in HA parameters among HF stages}

As summarized in Table 3 and Figure 2, a significant difference in the median metric was only found between 

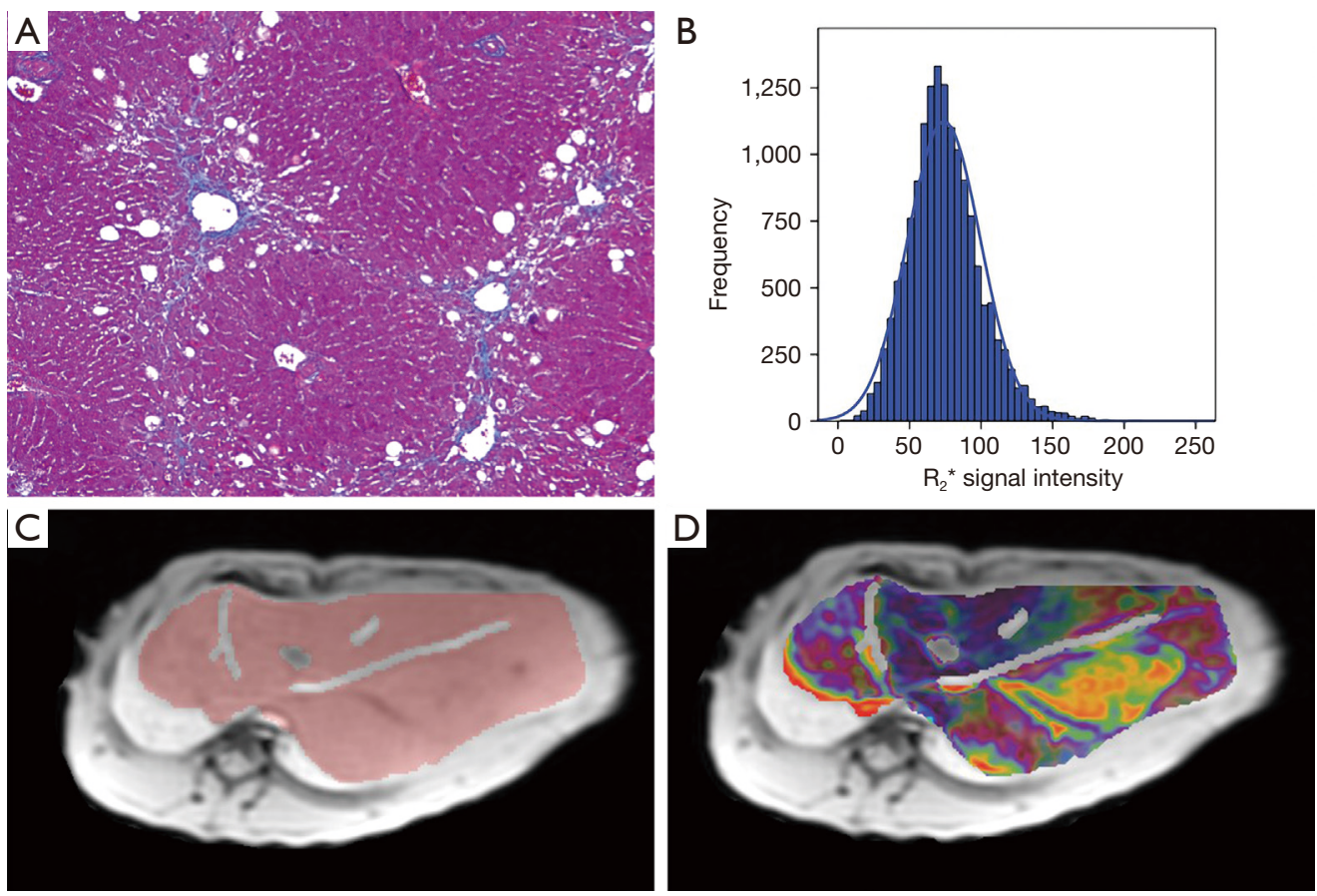

Figure 1 Typical figure of pathology, histogram analysis, ROI and rainbow $\mathrm{R}_{2}{ }^{*}$ map. (A) Pathological image (Masson's trichrome slices, $\mathrm{F}$ $\times 100$ ) for stage F2: portal fibrosis and a few septa. (B) Data acquired from each slice were summed to derive the voxel-by-voxel $R_{2}{ }^{*}$ values for the whole liver, and the $\mathrm{R}_{2}{ }^{*}$ histogram images were generated for stage F2; (C) schematic drawing of ROI; (D) rainbow $\mathrm{R}_{2}{ }^{*}$ map.

F0 and F4. When comparing all HF stage pairs, with the exception of F0 vs. F1 and F1 vs. F2, a significant difference was observed in $75^{\text {th }}(\mathrm{P}<0.05)$. Similarly, 90 ${ }^{\text {th }}$ value was significantly different among all LF pairs compared $(\mathrm{P}<0.05)$, except $\mathrm{F} 0$ vs. $\mathrm{F} 1$ and $\mathrm{F} 2$ vs. $\mathrm{F} 3$. A significant difference in entropy was found between $\mathrm{F} 4$ and (F1-F3) $(\mathrm{P}<0.05)$. Moreover, the inhomogeneity value did not significantly discriminate $\mathrm{F} 1$ vs. F2, F2 vs. F3 and F3 vs. F4 $(\mathrm{P}>0.05)$. Additionally, a significant difference in entropy was found between F4 and $(\mathrm{F} 1-\mathrm{F} 3)(\mathrm{P}<0.05)$.

\section{ROC curve analyses}

Among the five HA parameters that increased with HF progression, ROC curve analysis revealed the best diagnostic value for the $75^{\text {th }}$ percentile (AUC $=0.86,0.87$, 0.97 and 0.86 ), followed by $90^{\text {th }}$ percentile (AUC $=0.88$, $0.87,0.80$ and 0.82 ) and inhomogeneity ( $\mathrm{AUC}=0.83$, $0.83,0.83$ and 0.82 ), which was higher than the entropy (AUC $=0.65,0.65,0.70$ and 0.71) and median (AUC $=0.65$, $0.62,0.60$ and 0.61 ) for staging $\geq F 1, \geq F 2, \geq F 3, \geq F 4$, respectively, as presented in Table 3 and Figure 3.

\section{Discussion}

In this animal study, we investigated the diagnostic value of BOLD-fMRI in diagnosing and staging HF from the perspective of whole-liver HA using histopathological results as the reference standard. As shown in our study, the histogram-derived parameters including the median, inhomogeneity, entropy, and the $75^{\text {th }}$ and $90^{\text {th }}$ percentiles increased progressively with increasing HF stages, suggesting that BOLD-fMRI offers a robust method that may improve the assessment of HF.

As HF progresses, intrahepatic vasoconstriction, extracellular matrix deposition, and regeneration nodule formation occur, contributing to increased portal venous pressure and therefore a lower partial oxygen level (16). Previous studies have reported that a decreased ratio of oxygenated hemoglobin to deoxyhemoglobin reduces microscopic field gradients and decrease $\mathrm{T}_{2}{ }^{*}$ relaxation time, thus $\mathrm{R}_{2}{ }^{*}\left(1 / \mathrm{T}_{2}{ }^{*}\right)$ map can be an effective imaging biomarker of HF processes $(17,18)$. Studies in growing numbers have investigated the HA or deep learning of MRI functional sequences, including the hepatobiliary phase (19) 


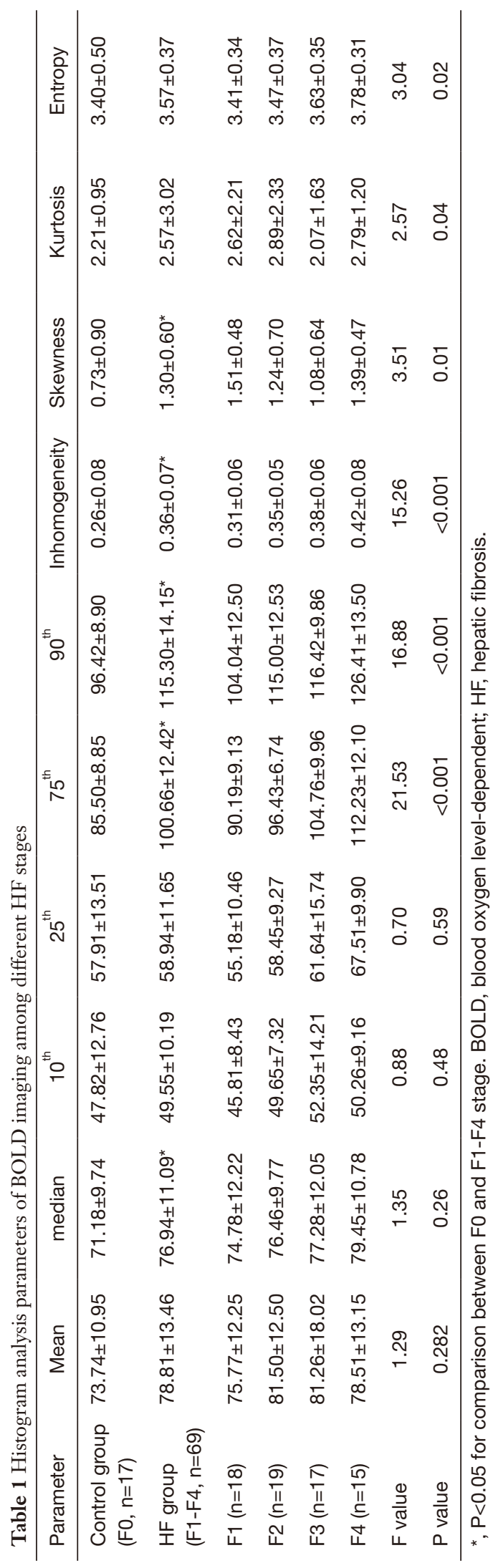

Table 2 Correlation between histogram parameters and HF stage

\begin{tabular}{lccc}
\hline Parameter & $\mathrm{r}$ & $95 \% \mathrm{Cl}$ & $\mathrm{P}$ \\
\hline Mean & 0.204 & $0.022-0.396$ & 0.063 \\
Median & 0.226 & $0.043-0.426$ & $0.038^{*}$ \\
$10^{\text {th }}$ & 0.150 & $-0.061-0.380$ & 0.173 \\
$25^{\text {th }}$ & 0.133 & $-0.075-0.348$ & 0.229 \\
$75^{\text {th }}$ & 0.718 & $0.592-0.804$ & $<0.001^{*}$ \\
$90^{\text {th }}$ & 0.670 & $0.544-0.772$ & $<0.001^{*}$ \\
Inhomogeneity & 0.644 & $0.462-0.774$ & $<0.001^{*}$ \\
Skewness & 0.157 & $-0.058-0.368$ & 0.155 \\
Kurtosis & 0.152 & $-0.041-0.337$ & 0.167 \\
Entropy & 0.318 & $0.110-0.494$ & $0.003^{*}$ \\
\hline
\end{tabular}

* $\mathrm{P}<0.05$. HF, hepatic fibrosis.

and proton density (20), in staging HF. However, these studies drew the ROI around partial liver tissue or in one slice of the liver. These ROI selections were subjective and easily led to sampling bias because the grayscalelevel information for the whole liver cannot be fully used. Thus, to improve the reliability and reproducibility of HA parameters, another purpose of this research was to explore the diagnostic accuracy of whole-liver HA of BOLD images in assessing grayscale heterogeneity and diagnosing HF.

Among the $\mathrm{n}^{\text {th }}$ percentile metrics, the $75^{\text {th }}$ percentile demonstrated the highest value in predicting HF severity, followed by the $90^{\text {th }}$ percentile, whereas the lower percentiles were of little use. This revealed that higher percentiles are more valuable in distinguishing HF. The signal intensity of the $75^{\text {th }}$ percentile is smaller than the value acquired by excluding the $25 \%$ maximum values, which may reflect incorrect signal intensity from image noise or artifacts that were included in ROIs. Thus, the $75^{\text {th }}$ percentile was more representative of pathological characteristics of the liver parenchyma. Zheng et al. (12) also suggested higher percentiles were more helpful in staging HF with apparent diffusion coefficient (ADC) HA in humans. The $90^{\text {th }}$ percentile was superior to the $75^{\text {th }}$ percentile in their study, possibly because the BOLD image is more susceptible to noise variations (21) and animal models have more breathing and movement artifacts. Compared with the median value, the mean value did not significantly discriminate HF stages, which was in agreement with the finding of $\mathrm{Hu}$ et al. (22). This is presumably because the robustness of the median value 

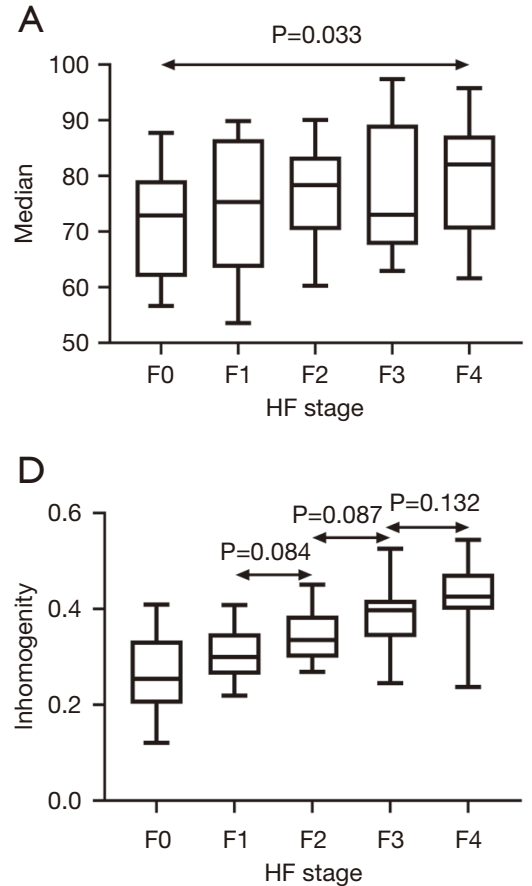

B

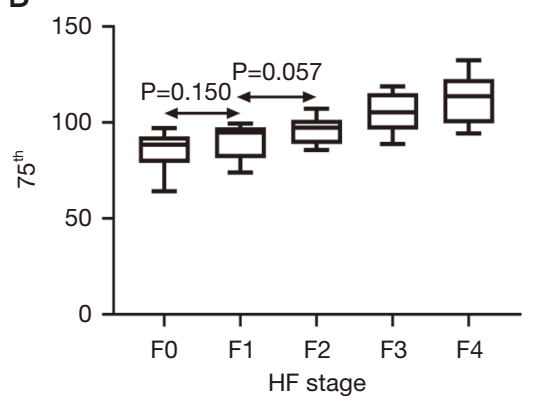

$E$

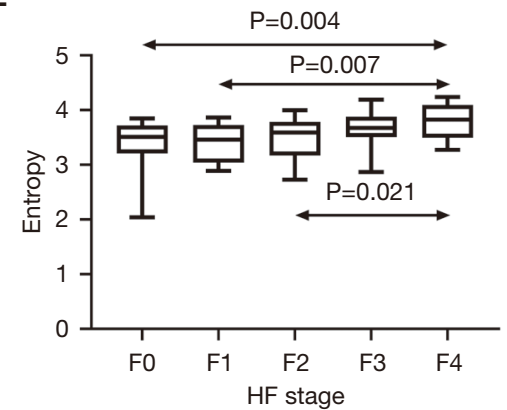

C

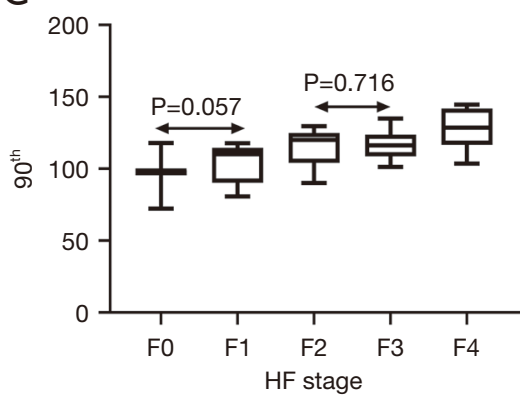

Figure 2 Boxplot of median (A), $75^{\text {th }}$ percentile (B), $90^{\text {th }}$ percentile (C), inhomogeneity (D) and entropy (E) values with HF ranging from F0 to F4. HF, hepatic fibrosis.

calculation was easily affected by outlier pixels at the boundaries of the liver or noise.

Our study revealed that the inhomogeneity parameter of the $\mathrm{R}_{2}{ }^{*}$ map had a higher correlation and diagnostic value, indicating inhomogeneity may be a potential biomarker reflecting increased heterogeneity for staging $\mathrm{HF}$, which was consistent with the conclusion of Kim et al. (23). Their study suggested that the coefficient of variation from GdEOB-DTPA-enhanced MRI hepatobiliary phase was a meaningful parameter in predicting the fibrosis grade. Similarly, Yang et al. (13) reported that variance from SWI in advanced HF was greater than normal hepatic tissue or early $\mathrm{HF}$, and it proved to be valuable in predicting the severity of HF. Increased deoxyhemoglobin with more nonhomogeneous iron deposition, disrupted architecture with narrowed sinusoids, more stenosis and edema, and increased extracellular collagen deposition $(1,24)$ contribute to increased grayscale heterogeneity and consequent changes in the inhomogeneity parameter.

In our study, no significant correlation with HF progression was found for skewness, which agrees with the study of Sheng et al. (25). This indicates that the distribution of the $\mathrm{R}_{2}{ }^{*}$ grayscale intensity is less skewed for liver parenchyma and therefore was insufficient at detecting HF. In previous studies, Yoon et al. (26) and Cannella (27) demonstrated that kurtosis, the measurement of peakedness, was a good discriminative feature for differentiating cirrhosis from normal liver tissue and predicting liver function, which was contrary to our study conclusion. Different ROI selection methods may account for this result. ROI was only drawn within one slice in their study, the selection of a regional region may be subjective and easily result in sampling bias. But, in our study, ROIs were drawn based on the whole liver. This method may be more reliable and increase the stability of parameter measurements. The entropy feature identifies the randomness and complexity in the $\mathrm{HA}$ of $\mathrm{R}_{2}{ }^{*}$ value of the whole-liver, and a higher entropy value corresponds to more irregular oxygenated hemoglobin or iron deposition existing throughout the entirety of the liver parenchyma. Both Fujimoto et al. (28) and Yu et al. (29) found that the demonstrated that entropy metric increased with increasing HF stage, and accurate in detecting HF, which was similar to our study conclusion.

There are several limitations to our study. First, the pathological results of liver were heterogeneous due to fat 
Table 3 ROC analysis of histogram analysis for staging HF

\begin{tabular}{|c|c|c|c|c|}
\hline Parameters & F0 vs. F1-F4 ( $\geq F 1)$ & F0-1 vs. F2-F4 ( $\geq F 2$ ) & F0-2 vs. F3-F4 ( $\geq$ F3) & F0-3 vs. F4 ( $\geq$ F4) \\
\hline \multicolumn{5}{|l|}{ Median } \\
\hline AUC (95\% Cl) & $0.65(0.54-0.75)$ & $0.62(0.50-0.72)$ & $0.60(0.48-0.70)$ & $0.61(0.50-0.72)$ \\
\hline Cut-off value & 80.87 & 65.05 & 65.05 & 81.60 \\
\hline Sensitivity (95\% Cl) & $40.91(29.0-53.7)$ & 88.05 (75.7-95.5) & 93.75 (79.9-99.2) & $53.33(26.6-78.7)$ \\
\hline Youden Index & 0.30 & 0.23 & 0.25 & 0.24 \\
\hline \multicolumn{5}{|l|}{$75^{\text {th }}$} \\
\hline AUC (95\% Cl) & $0.86(0.77-0.93)$ & $0.87(0.78-0.93)$ & $0.87(0.78-0.93)$ & $0.86(0.77-0.93)$ \\
\hline Cut-off value & 93.22 & 99.48 & 103.17 & 107.14 \\
\hline Youden Index & 0.66 & 0.60 & 0.59 & 0.63 \\
\hline \multicolumn{5}{|l|}{$90^{\text {th }}$} \\
\hline AUC $(95 \% \mathrm{Cl})$ & $0.88(0.79-0.94)$ & $0.87(0.78-0.93)$ & $0.80(0.70-0.88)$ & $0.82(0.73-0.90)$ \\
\hline Cut-off value & 99.11 & 101.90 & 100.28 & 116.25 \\
\hline Sensitivity (95\% Cl) & 86.36 (75.7-93.6) & 90.00 (78.2-96.7) & $100.00(89.1-100.0)$ & $80.00(51.9-95.7)$ \\
\hline Specificity $(95 \% \mathrm{Cl})$ & 94.44 (72.7-99.9) & 70.59 (52.5-84.9) & $51.92(37.6-66.0)$ & $69.57(57.3-80.1)$ \\
\hline Youden Index & 0.81 & 0.61 & 0.52 & 0.50 \\
\hline \multicolumn{5}{|l|}{ Inhomogeneity } \\
\hline Youden index & 0.58 & 0.54 & 0.58 & 0.58 \\
\hline \multicolumn{5}{|l|}{ Entropy } \\
\hline AUC (95\% Cl) & $0.65(0.54-0.75)$ & $0.65(0.54-0.75)$ & $0.70(0.58-0.79)$ & $0.71(0.60-0.80)$ \\
\hline Cut-off value & 3.85 & 3.47 & 3.47 & 3.99 \\
\hline Sensitivity (95\% Cl) & $21.21(12.1-33.0)$ & $74.00(59.7-85.4)$ & 81.25 (63.6-92.8) & $40.00(16.3-67.7)$ \\
\hline Specificity $(95 \% \mathrm{Cl})$ & $100.00(81.5-100.0)$ & $52.94(35.1-70.2)$ & $48.08(34.0-62.4)$ & 98.55 (92.2-100.0) \\
\hline Youden index & 0.21 & 0.27 & 0.29 & 0.39 \\
\hline
\end{tabular}

HF, hepatic fibrosis; ROC, receiver operating characteristic; AUC, area under ROC curve.

and iron deposition, necrotic inflammation and steatosis (30-32). These pathological process were the main heterogeneity factors associated with the inhomogeneity result, but they cannot be measured with common Masson's trichrome staining. Therefore, further studies are required to confirm the robustness of BOLD-fMRI in differentiating fibrosis stages, with corrections performed for iron and fat deposition, inflammation. Second, $\mathrm{CCl}_{4}$-induced rabbit 
A

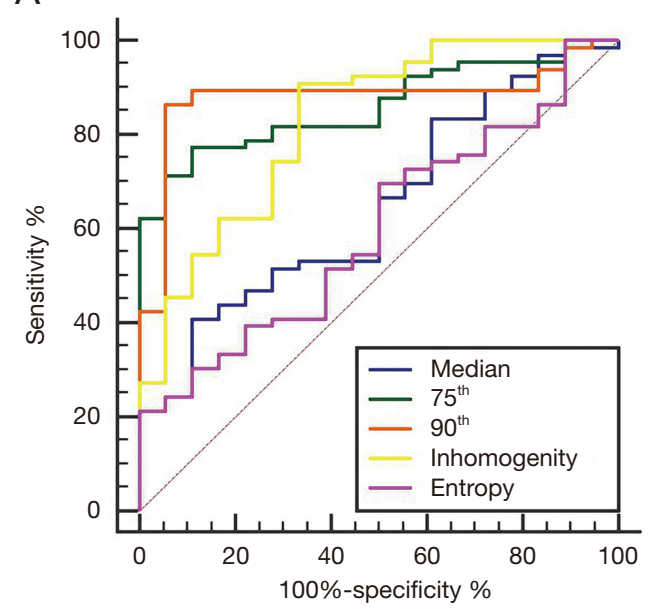

C

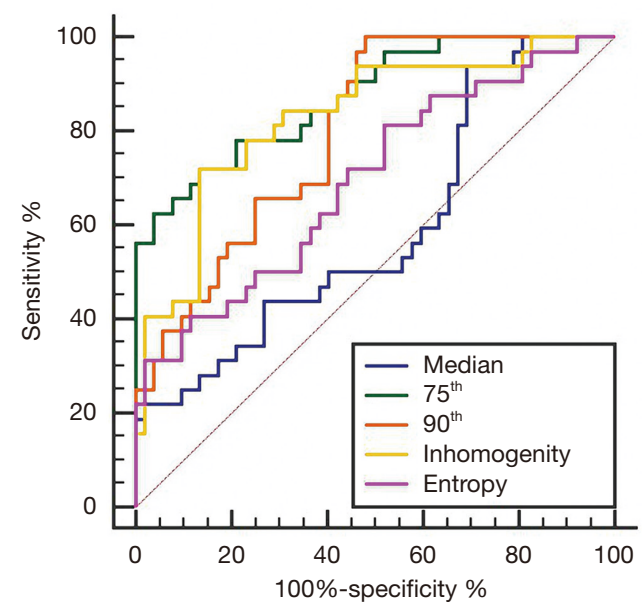

B

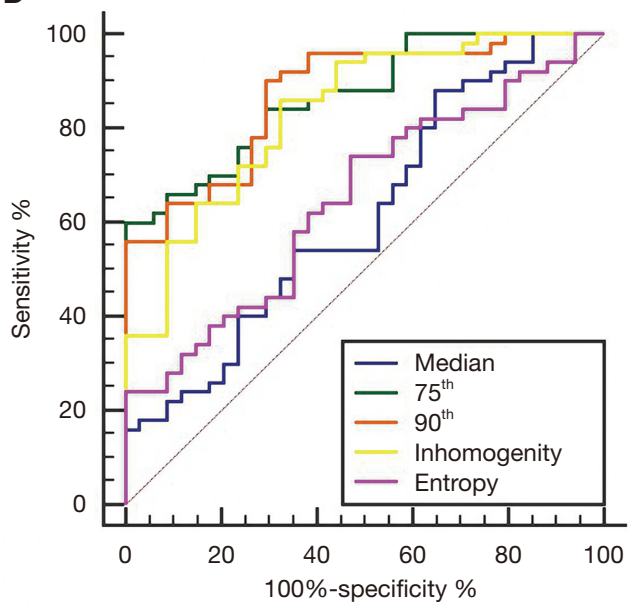

D

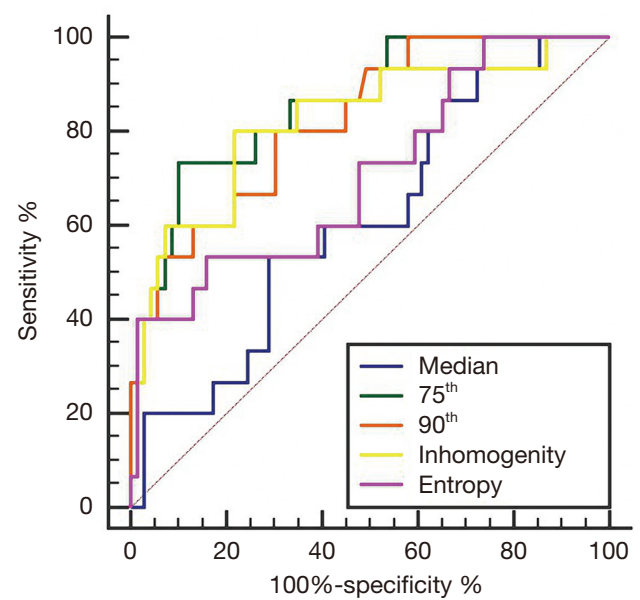

Figure 3 Receiver operating characteristic curves analyzing the ability of median, $75^{\text {th }}$ percentile, $90^{\text {th }}$ percentile, inhomogeneity and entropy in identifying HF stage $\geq F 1(A), \geq F 2(B), \geq F 3(C)$ and $\geq F 4(D)$, respectively. HF, hepatic fibrosis.

model may not precisely present the pathological results in the human liver; thus, subsequent researches in the human setting were needed to prove the conclusions of our study.

\section{Conclusions}

Whole-liver HA of BOLD-fMRI can objectively assess the grayscale heterogeneity of the entire liver. It provides higher diagnostic value in diagnosing HF stages, supporting its application as a promising noninvasive tool of $\mathrm{HF}$ in the future.

\section{Acknowledgments}

Funding: This work was supported by the National Natural
Science Foundation of China (81771798; 81771805 ; 81901696), Youth Project of Changzhou City Health Commission (QN2020022) and Jiangsu Key Research and Development Project (EB2018646).

\section{Footnote}

Reporting Checklist: The authors have completed the ARRIVE reporting checklist. Available at http://dx.doi. org/10.21037/apm-20-1753

Data Sharing Statement: Available at http://dx.doi. org/10.21037/apm-20-1753

Conflicts of Interest: All authors have completed the ICMJE 
uniform disclosure form (available at http://dx.doi. org/10.21037/apm-20-1753). The authors have no conflicts of interest to declare.

Ethical Statement: The authors are accountable for all aspects of the work in ensuring that questions related to the accuracy or integrity of any part of the work are appropriately investigated and resolved. Experiments were performed under a project license (No. 2019-022) granted by institutional ethics board of Third Affiliated Hospital of Soochow University, in compliance with NIH guidelines for the care and use of animals.

Open Access Statement: This is an Open Access article distributed in accordance with the Creative Commons Attribution-NonCommercial-NoDerivs 4.0 International License (CC BY-NC-ND 4.0), which permits the noncommercial replication and distribution of the article with the strict proviso that no changes or edits are made and the original work is properly cited (including links to both the formal publication through the relevant DOI and the license). See: https://creativecommons.org/licenses/by-nc-nd/4.0/.

\section{References}

1. Tacke F, Trautwein C. Mechanisms of liver fibrosis resolution. J Hepatol 2015;63:1038-9.

2. Hernandez-Gea V, Friedman SL. Pathogenesis of liver fibrosis. Annu Rev Pathol 2011;6:425-56.

3. Tacke F, Weiskirchen R. An update on the recent advances in antifibrotic therapy. Expert Rev Gastroenterol Hepatol 2018;12:1143-52.

4. Schuppan D, Ashfaq-Khan M, Yang AT, et al. Liver fibrosis: Direct antifibrotic agents and targeted therapies. Matrix Biol 2018;68-69:435-51.

5. Altamirano-Barrera A, Barranco-Fragoso B, MéndezSánchez N. Management strategies for liver fibrosis. Ann Hepatol 2017;16:48-56.

6. Czeczok TW, Van Arnam JS, Wood LD, et al. The Almost-Normal Liver Biopsy: Presentation, Clinical Associations, and Outcome. Am J Surg Pathol 2017;41:1247-53.

7. Rockey DC, Caldwell SH, Goodman ZD, et al. Liver biopsy. Hepatology 2009;49:1017-44.

8. Xu XY, Wang WS, Zhang QM, et al. Performance of common imaging techniques vs serum biomarkers in assessing fibrosis in patients with chronic hepatitis B: A systematic review and meta-analysis. World J Clin Cases
2019;7:2022-37.

9. Patterson AJ, Priest AN, Bowden DJ, et al. Quantitative BOLD imaging at $3 \mathrm{~T}$ : Temporal changes in hepatocellular carcinoma and fibrosis following oxygen challenge. J Magn Reson Imaging 2016;44:739-44.

10. Zhan Y, Wu Y, Chen J. Carbogen gas-challenge BOLD fMRI in assessment of liver hypoxia after portal microcapsules implantation. PLoS One 2019;14:e0225665.

11. Jin N, Deng J, Chadashvili T, et al. Carbogen gaschallenge BOLD MR imaging in a rat model of diethylnitrosamine-induced liver fibrosis. Radiology 2010;254:129-37.

12. Zheng Y, Xu YS, Liu Z, et al. Whole-Liver Apparent Diffusion Coefficient Histogram Analysis for the Diagnosis and Staging of Liver Fibrosis. J Magn Reson Imaging 2020;51:1745-54.

13. Yang ZX, Liang HY, Hu XX, et al. Feasibility of histogram analysis of susceptibility-weighted MRI for staging of liver fibrosis. Diagn Interv Radiol 2016;22:301-7.

14. Schawkat K, Ciritsis A, von Ulmenstein S, et al. Diagnostic accuracy of texture analysis and machine learning for quantification of liver fibrosis in MRI: correlation with MR elastography and histopathology. Eur Radiol 2020;30:4675-85

15. Zou LQ, Zhao F, Zhang H, et al. Staging liver fibrosis on multiparametric MRI in a rabbit model with elastography, susceptibility-weighted imaging and T1 $\rho$ imaging: a preliminary study. Acta Radiol 2021;62:155-63.

16. Turco L, Garcia-Tsao G. Portal Hypertension: Pathogenesis and Diagnosis. Clin Liver Dis 2019;23:573-87.

17. Bane O, Besa C, Wagner M, et al. Feasibility and reproducibility of BOLD and TOLD measurements in the liver with oxygen and carbogen gas challenge in healthy volunteers and patients with hepatocellular carcinoma. J Magn Reson Imaging 2016;43:866-76.

18. Yuan F, Song B, Huang Z, et al. Oxygen and Glucose as Stimulation Agents for BOLD Functional MR Imaging of Rabbit Liver: A Feasibility Study. Magn Reson Med Sci 2018;17:145-50.

19. Asayama $Y$, Nishie A, Ishigami $K$, et al. Histogram analysis of noncancerous liver parenchyma on gadoxetic acidenhanced MRI: predictive value for liver function and pathology. Abdom Radiol (NY) 2016;41:1751-7.

20. Yu H, Buch K, Li B, et al. Utility of texture analysis for quantifying hepatic fibrosis on proton density MRI. J Magn Reson Imaging 2015;42:1259-65.

21. Han S, Son JP, Cho H, et al. Gradient-echo and spin- 
echo blood oxygenation level-dependent functional MRI at ultrahigh fields of 9.4 and 15.2 Tesla. Magn Reson Med 2019;81:1237-46.

22. Hu F, Yang R, Huang $Z$, et al. Liver fibrosis: in vivo evaluation using intravoxel incoherent motion-derived histogram metrics with histopathologic findings at 3.0 T. Abdom Radiol (NY) 2017;42:2855-63.

23. Kim H, Park SH, Kim EK, et al. Histogram analysis of gadoxetic acid-enhanced MRI for quantitative hepatic fibrosis measurement. PloS one 2014;9:e114224.

24. Seki E, Brenner DA. Recent advancement of molecular mechanisms of liver fibrosis. J Hepatobiliary Pancreat Sci 2015;22:512-8.

25. Sheng RF, Jin KP, Yang L, et al. Histogram Analysis of Diffusion Kurtosis Magnetic Resonance Imaging for Diagnosis of Hepatic Fibrosis. Korean J Radiol 2018;19:916-22.

26. Yoon JH, Lee JM, Kim E, et al. Quantitative Liver Function Analysis: Volumetric T1 Mapping with Fast Multisection B(1) Inhomogeneity Correction in Hepatocyte-specific Contrast-enhanced Liver MR

Cite this article as: Liu HF, Wang Q, Du YN, Ding JL, Zhang JG, Xing W. Whole-liver histogram analysis of blood oxygen level-dependent functional magnetic resonance imaging in evaluating hepatic fibrosis. Ann Palliat Med 2021;10(3):2567-2576. doi: 10.21037/apm-20-1753
Imaging. Radiology 2017;282:408-17.

27. Cannella R, Borhani AA, Tublin M, et al. Diagnostic value of MR-based texture analysis for the assessment of hepatic fibrosis in patients with nonalcoholic fatty liver disease (NAFLD). Abdom Radiol (NY) 2019;44:1816-24.

28. Fujimoto K, Tonan T, Azuma S, et al. Evaluation of the mean and entropy of apparent diffusion coefficient values in chronic hepatitis $\mathrm{C}$ : correlation with pathologic fibrosis stage and inflammatory activity grade. Radiology 2011;258:739-48.

29. Yu H, Touret AS, Li B, et al. Application of texture analysis on parametric $\mathrm{T}(1)$ and $\mathrm{T}(2)$ maps for detection of hepatic fibrosis. J Magn Reson Imaging 2017;45:250-9.

30. Yukinori Koyama, David A Brenner. Liver inflammation and fibrosis. J Clin Invest 2017;127:55-64.

31. Mehta KJ, Farnaud SJ, Sharp PA. Iron and liver fibrosis: Mechanistic and clinical aspects. World J Gastroenterol 2019;25:521-38.

32. Karanjia RN, Crossey MM, Cox IJ. Hepatic steatosis and fibrosis: Non-invasive assessment. World J Gastroenterol 2016;22:9880-97. 